\title{
Impact of a New Cost-Effectiveness Threshold Implementation on Cancer Formulary Decisions in Jordan
}

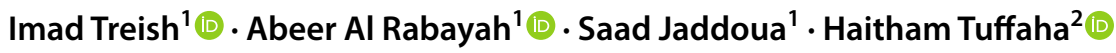

Accepted: 30 June 2021 / Published online: 13 November 2021

(c) The Author(s) 2021

\begin{abstract}
Rising prices of novel cancer medications are increasing the economic burden from cancer in Jordan, risking the ability of cancer patients to access lifesaving and life-extending treatments. Furthermore, in the absence of a national health technology assessment (HTA) framework, medication prices in Jordan are set based on manufacturers' pricing considerations and not a value proposition. In response to these challenges, King Hussein Cancer Center (KHCC), the de facto national cancer institute, developed a first-in-country, cancer-specific, cost-effectiveness threshold (CET) to aid institutional decision makers in approving only cost-effective medications. Over the past 10 years, cost-effectiveness analyses based on this CET have led to the introduction of $>70 \%$ of requested novel cancer medications after manufacturers agreed to lower prices, beyond registration prices, to meet the CET. Future work is warranted to empirically derive a CET for Jordan to better guide reimbursement decisions.
\end{abstract}

\section{Key Points for Decision Makers}

We describe the development and application of a costeffectiveness threshold (CET) at a comprehensive cancer center in Jordan.

The application of the CET threshold in the health technology assessment of new cancer drugs resulted in significant reductions in acquisition costs.

Future work is needed to empirically derive a countryspecific CET.

Abeer Al Rabayah

ARabaiah@KHCC.JO

Imad Treish

itreish@khcc.jo

Haitham Tuffaha

h.tuffaha@uq.edu.au

1 Department of Pharmacy, Center for Drug Policy and Technology Assessment (CDPTA), King Hussein Cancer Center, Amman, Jordan

2 Centre for the Business and Economics of Health, The University of Queensland, Brisbane, QLD 4067, Australia

\section{Introduction}

The economic burden of cancer has been increasing in Jordan similar to other countries in the world [1]. In Jordan, this increase is fueled by an increase in the prices of novel cancer medications $[2,3]$ and an increase in cancer incidence (5556 new cancer cases in 2015 vs 3670 in 2005) [4]. The main driver for cancer incidence increase is the near-doubling of the population (9.53 million in 2015 vs 5.48 million in 2005) [5]. Additionally, the incidence rate of cancer has also increased (age-standardized incidence rate [ASIR] 113/100,000 in 2005 vs 118/100,000 in 2015), which is attributed to aging (life expectancy at birth was 72.9 in 2007 vs 74.3 in 2017), environmental exposure and lifestyle changes [4-6].

The World Bank classifies Jordan as an upper-middleincome economy country [7]. The gross domestic production national income (GDP) per capita grew from US $\$ 3398$ in 2008 to US $\$ 40,163$ in 2017 [8]. Total health care expenditure (THE) in Jordan has doubled in the last decade, reaching US\$3.62 billion in 2017 [8]. Moreover, the per-capita THE has increased from 177.50 Jordanian dinar (JD) to JD255.00 in the same period. Jordan's THE is higher than similar-income countries in the region (9\% vs 5\% of GDP), leading to improved access to health care services such as cancer care. A 1999 royal decree ensured that all Jordanians diagnosed with cancer receive cancer 
treatment at no cost in the public-sector hospitals and the King Hussein Cancer Center (KHCC) [9].

KHCC was established in 2002 to provide much-needed comprehensive care for cancer patients in Jordan and the region [10]. Care is delivered using a multidisciplinary approach based on institutional clinical practice guidelines (CPGs) adapted from various international guidelines [9]. Furthermore, KHCC implemented a robust formulary management system to provide safe and cost-effective medications for cancer patients under the auspices of the Pharmacy \& Therapeutics Committee (P\&T). Pharmacy expenditure, mostly on cancer medications, grew by $63 \%$ from 2005 to 2008. In 2006, the P\&T became concerned that the increasing rate of novel cancer medicines' introduction to the market would lead to detrimental financial consequences for KHCC and the national health budget if local pharmacoeconomic metrics were not considered in the evaluation of new medication additions. Conversely, excluding such medications from the formulary would limit patients' access to life-saving and life-prolonging medications. Unfortunately, Jordan did not have a health technology assessment (HTA) framework to guide decision makers in approving new health interventions [11].

Public (selling) prices of medications in Jordan are set by the Jordan Food and Drug Administration (JFDA) under the External Reference Pricing (ERP) Policy [12]. The set price should be the lowest of the reference prices (benchmarks): the country-of-origin price, the reference basket median price, the manufacturer export price, and Saudi Arabia's public price. Pharmacoeconomic evidence has a limited impact on pricing decisions [13]. The public price is applied in community pharmacies and private hospitals for self-paying patients. Private insurance companies typically apply a contractual discount to the public price. Reimbursement rates in the public-sector hospitals are based on acquisition costs of the Joint Procurement Department (JPD), which initiates public tenders to purchase medications that are on the Rational Drug List (RDL) on behalf of the public-sector hospitals. The RDL represents Jordan's national formulary and it is an output of the work of both technical and national committees that represent all related health sectors. However, costeffectiveness analysis (CEA) does not impact the listing decision of new medications to the RDL $[11,13]$.

Although the government has not set a specific budget for cancer care, KHCC sought to implement an HTA framework to ensure that only cost-effective medications are added to its formulary, therefore attempting to strike a balance between ensuring access to new medications and the increasing expenditure on medications. Furthermore, the framework would empower KHCC to directly negotiate medication costs with pharmaceutical companies in a more objective and transparent way. Lastly, an HTA framework would serve not only KHCC but also other providers in the country due to KHCC's professional influence over other institutions.

The objective of this paper is to describe the development of a cancer-specific cost-effectiveness threshold (CET) for the first time in Jordan and the impact of this CET on formulary decisions related to novel cancer medications at KHCC.

\section{Cost-Effectiveness Threshold (CET) Development and Application}

\subsection{Overview}

KHCC considered several options for setting the CET. One option was to adopt the World Health Organization (WHO) recommendation of considering interventions with an incremental cost-effectiveness ratio (ICER) of $<3 \times$ GDP per capita/disability-adjusted life-year (DALY) to be cost effective [14]. However, this approach produced a CET of US\$7539 (based on 2006 data), which would not have allowed the introduction of most cancer therapies, thereby limiting access to life-saving or life-extending medications. Alternatively, the adoption of a CET from a developed economy would have overestimated the CET. This led KHCC to develop a CET based on local considerations. Given pharmacoeconomics expertise limitations and the absence of international guidelines/methods at that time to estimate thresholds based on the opportunity cost to our health budget, a decision was made to follow a precedent-based approach [15], whereby a CET is determined in relation to the cost effectiveness of an oncology medication that met the following criteria:

1. Novelty medication for cancer treatment to make our CET applicable to the new class of cancer-targeted therapies;

2. Considered by clinical experts as the standard of care based on its significant clinical benefit and established safety profile.

Imatinib (Glivec ${ }^{\mathrm{TM}}$ ) met the agreed-upon criteria. It was first approved in Jordan in 2003 for chronic myelogenous leukemia (CML). Despite the cost difference, imatinib immediately replaced interferon-based treatment for CML due to the dramatic improvement in outcome and the low side-effect profile [16]. At that time, only bone marrow transplantation was more expensive than imatinib, yet there was a clear consensus that imatinib should be the standard of care for CML. 


\subsection{Cost-Effectiveness Analysis of Imatinib}

A CEA was conducted from the perspective of a Jordanian health care system as a paying source. Details of the model were previously presented [17] and additional details are included in Appendix 1 (see electronic supplementary material). Briefly, a state transition (Markov) model was developed to estimate the expected costs and outcomes (life-years) of imatinib. The model included three health states: chronic phase CML, accelerated/blast phase CML, and death. Newly diagnosed patients with CML were expected to enter the model at the chronic phase. The mean starting age of CML patients was 50 years. Transition probabilities between health states were estimated based on the IRIS trial [16]. Mortality from non-cancer-related causes was estimated from the 2005 WHO life tables for Jordan. CML-related death was estimated from the IRIS trial [16].

The mean estimated survival with first-line treatment with imatinib for CML in the chronic phase was 11.59 life-years. Undiscounted lifetime costs were approximately US $\$ 500,690$ with imatinib. Imatinib, therefore, provided a threshold of approximately US $\$ 43,164 /$ life-year gained (LYG). In this model, and due to the relatively mild toxicity profile of imatinib compared with interferon, it was assumed that LYG from imatinib was considered an approximation for quality-adjusted life-year (QALY), as demonstrated by Reed and colleagues [18]. Henceforth, KHCC adopted a CET of US $\$ 43,000 /$ QALY.

In 2016, the KHCC Board of Trustees approved a modification to the 2006 threshold to account for inflation in the past 10 years. The newly approved CET would range between US $\$ 42,000$ and US $\$ 56,000$ per QALY. The upper end of this new range allowed for more flexibility when considering the approval of novel medications, whereas the lower end was used to negotiate the prices of new medications with similar entities that are already on the formulary.

\subsection{Health Technology Assessment (HTA) Pathway at KHCC and the Application of CET}

Effective 2006, all new formulary requests for novel cancer medications would be subjected to cost-effectiveness (CE) assessment. KHCC established the Center for Drug Policy and Evaluation (CDPTA) to conduct pharmacoeconomic analyses and conduct and appraise CE studies [19]. In 2012, a formulary submission pathway targeting pharmaceutical manufacturers was introduced under the formulary system policy. A CEA model would be either submitted by the pharmaceutical company as part of the HTA submission or developed internally. The CDPTA presents its CE assessment report to the P\&T. The report is based on a standardized template that addresses clinical pharmacology, clinical efficacy/effectiveness, safety, and economic evaluation along with expected resource utilization. The results of the adapted CE model, along with assessments of uncertainty and resource implications, are included in the economic evaluation section [19]. The P\&T may authorize the pharmacy department to initiate negotiations with the manufacturers to reduce acquisition costs in line with the CET.

\subsection{Impact of CET Application on Formulary Listing Decisions}

After obtaining institutional review board (IRB) approval, we conducted a review of $\mathrm{P} \& \mathrm{~T}$ records over the past 10 years to determine the impact of the new HTA framework on medication approvals.

CEAs of 30 anti-neoplastic medications were assessed from 2008 to 2018, including seven that were developed by the CDPTA staff. Of these, $22(73 \%)$ medications were added to the formulary, and eight were rejected. Ninety-three percent of listed medications were subject to financial risk sharing agreements with manufacturers to reach the KHCC threshold, whereas 7\% were listed based on the JFDA public price. Details are provided in Table 1.

\section{Discussion}

In the absence of national guidelines in Jordan, KHCC developed an HTA framework to ensure that only costeffective medications are added to its formulary. Based on unanimous consensus among KHCC and national medical experts supporting the use of first-line imatinib to treat $\mathrm{CML}$ in Jordan, we developed a CE model for imatinib in CML. The results of the model were used to set a CET that was adopted by KHCC's HTA framework.

Our approach to developing a CET is pragmatic in the sense that we have adopted a cancer-specific figure based on the cost effectiveness of imatinib mesylate in CML. To our knowledge, this is the first published work describing the development of a cancer-specific CET that has been successfully used for more than 10 years to assess the cost effectiveness of newly approved targeted and non-targeted treatments for cancer. Our approach of setting a CET based on the results of the decision modelling of imatinib in CML is analogous to using CEA of dialysis in end-stage renal disease as the basis for the CET figure most commonly cited in the US [20]. The principles that should be followed in choosing a reference drug include innovation (e.g., being a new class of drugs), significant clinical benefits (e.g., survival benefit relative to the standard of care), and safety [21]. Therefore, we believed that imatinib could be considered a reference drug against which the value for money of other cancer treatments could be compared [22, 23]. 


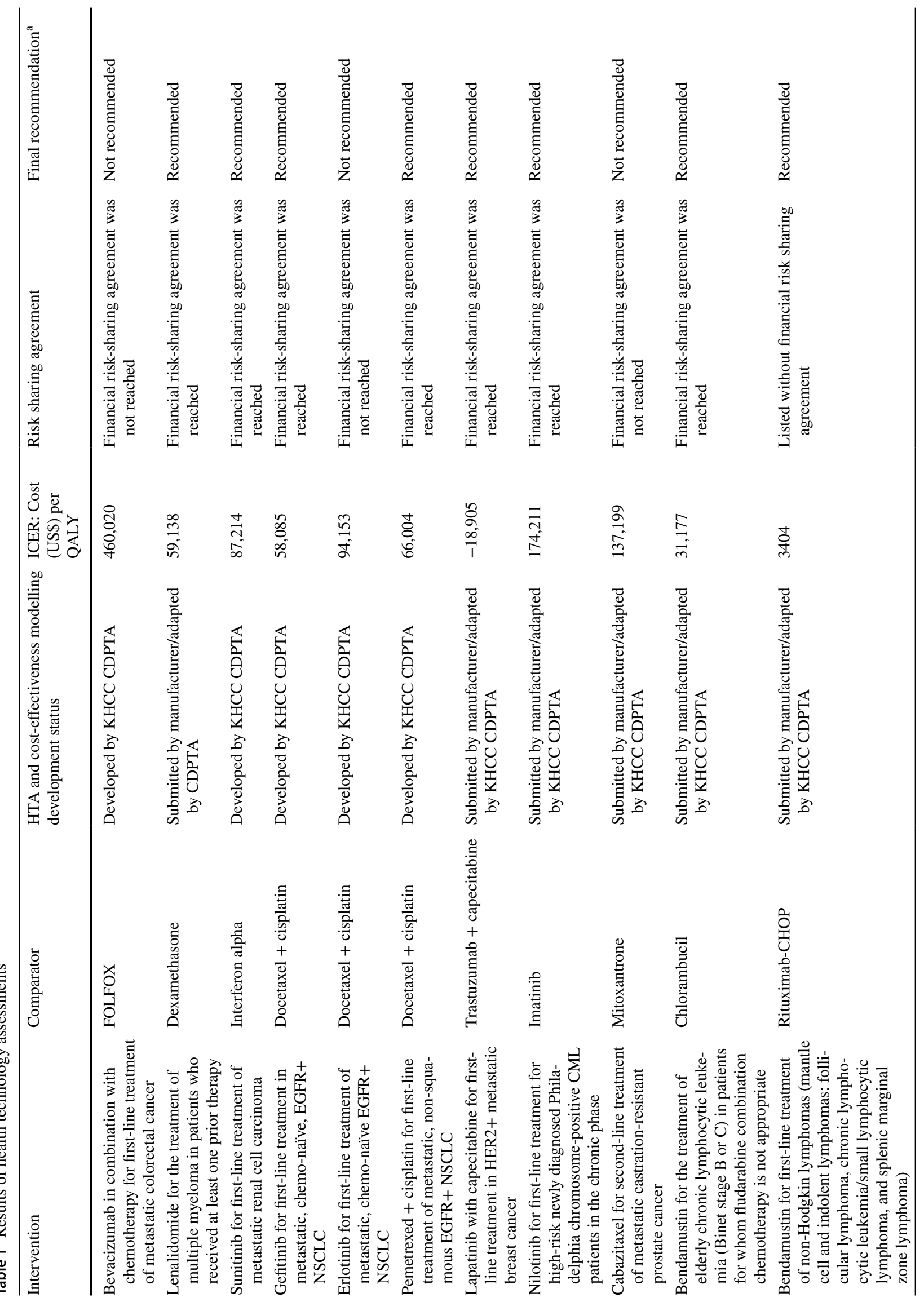




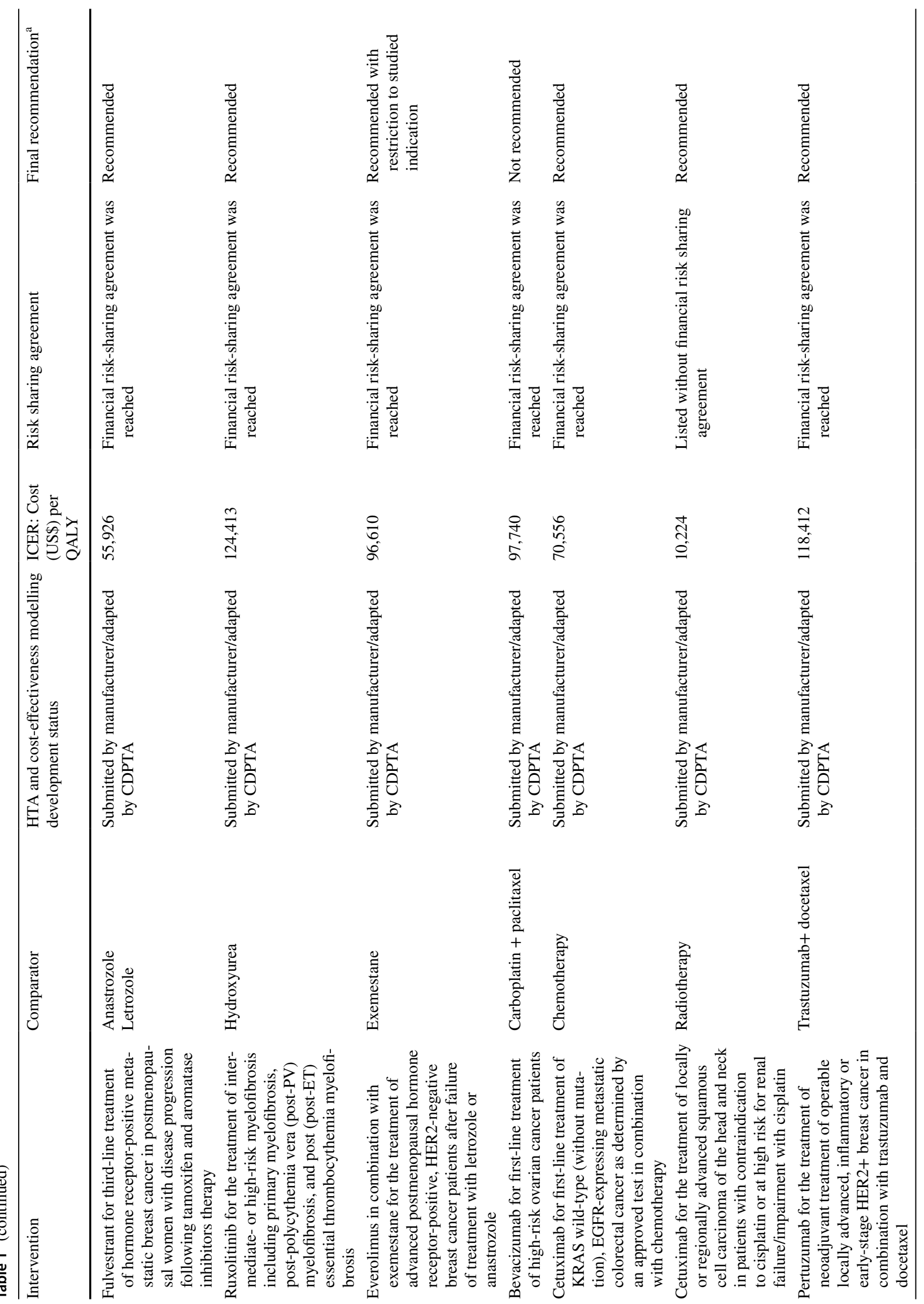




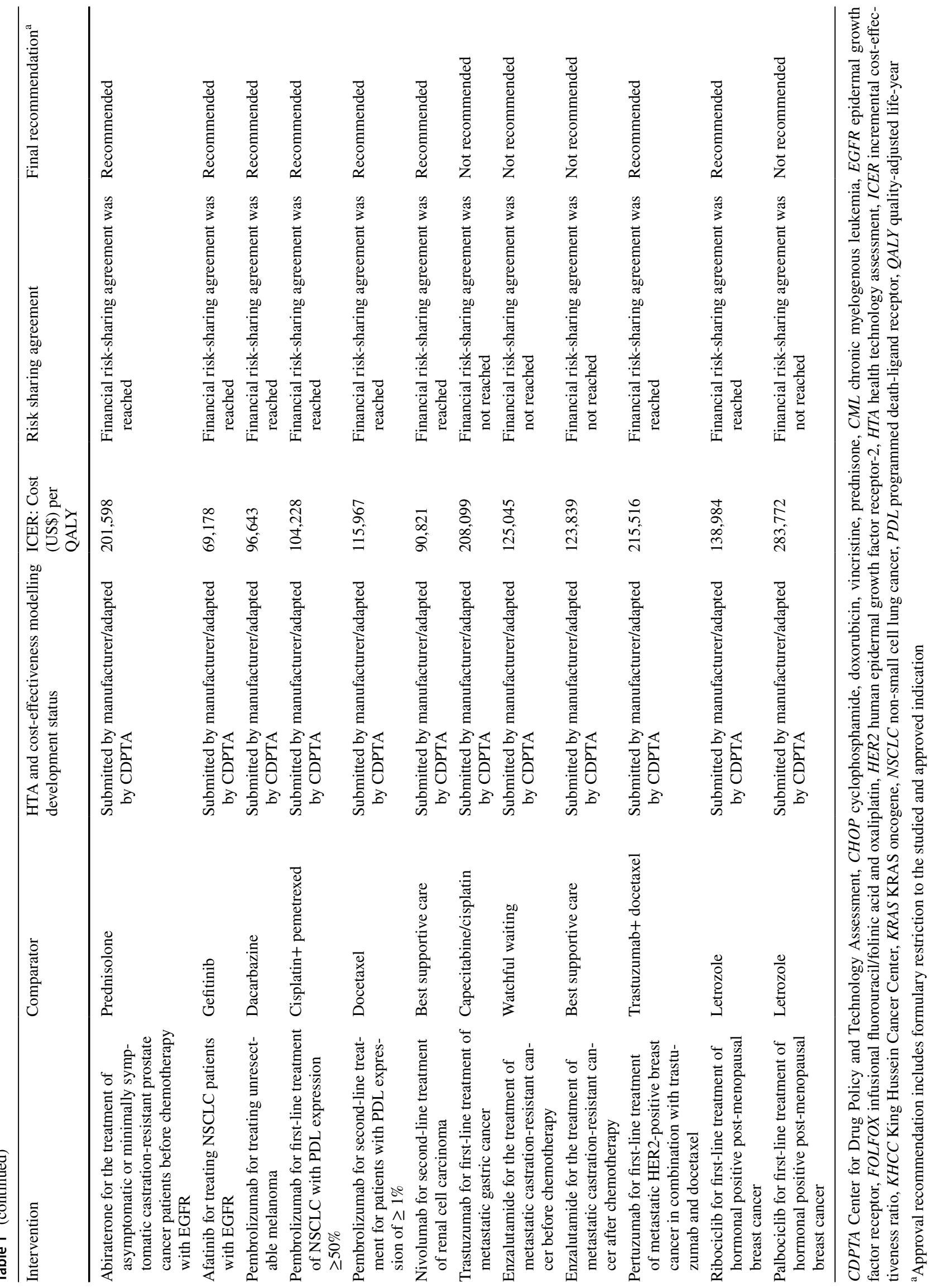


The establishment of the CET and the HTA framework provided us with a powerful and transparent tool to explain to our stakeholders (physicians, patients, and the pharmaceutical industry) how formulary decisions are made at KHCC. Furthermore, this approach effectively secured substantial discounts to bring the prices of medications in line with our CET or even better. Nonetheless, due to limited technical capacity and lack of expertise within KHCC at that time, limitations to our approach should be noted. The CET developed represents the average cost-effectiveness ratio (not the ICER), which is the ratio of the cost to benefit of an intervention without reference to a comparator. Moreover, we did not discount long-term costs and outcomes, and assumed a LYG equivalent to QALY gained. The latter assumptions were based on the work by Reed et al [18], which informed our analyses. Moreover, changing the threshold into a range was based on a Board decision due to increased societal pressures to increase access to new cancer treatments. In terms of the HTA framework overall impact, we did not develop clear indicators to comprehensively assess the outcomes of our decisions including overall savings to the Jordan healthcare system, impact on patient-reported outcomes, and impact on other institutions.

In a recent review of CETs worldwide, Santos and colleagues highlighted the lack of an empiric approach to determining the CET for most countries [15]. Existing CETs in many jurisdictions are based on either historic (e.g., NICE) or heuristic approaches (e.g., WHO threshold) that are not accurate as they can over- or underestimate the actual CET. Importantly, these CETs do not consider the opportunity cost from additional spending on the limited health budget and may lead to decisions that reduce rather than improve health gains. Similarly, CETs estimated based on studies of willingness to pay/accept (e.g., contingent valuation) tend to overestimate CET, and they also fail to account for the opportunity cost falling on the constrained health budgets. Despite its popularity, so much doubt has been cast on the genesis of NICE's CET figure [24]. In 2013, Claxton and colleagues developed a model for calculating CET based on opportunity cost using UK NHS data [25]. The estimated CET for the NHS was calculated to be US $\$ 18,609$ ( $£ 12,936) / Q A L Y$, which is much lower than the NICE threshold range. NICE has expressed its unwillingness to adopt this new CET as it would not allow for the entry of most new medications [26]. Interestingly, when this model was applied to estimate the CETs in several low- to middle-income countries (LMICs) based on their relative per-capita GDP and statistical life values, the CET for Jordan was between US\$1971 and US\$7757 per QALY [27]. Like NICE, Jordan would not adopt such a range as it would virtually eliminate most new cancer treatments. Thus, setting the CET too high may lead to inefficient budget allocation, whereas a CET that is too low would preclude the listing of novel health technologies.
Thus, a valid, reliable, and relevant CET remains elusive as policy makers around the world aim to strike a balance between improving access to new cancer treatments and maintaining budgetary controls; and between a CET that expresses the society's monetary valuation of health gains versus opportunity cost resulting from required disinvestment when adopting new technology. Future efforts, therefore, should be directed toward deriving a country-specific CET for Jordan based on the opportunity cost by considering marginal health spending and health gains. It is also essential to develop a national HTA framework that is responsive to stakeholders' needs in an equitable way $[28,29]$. Such a framework should be supported by practical but rigorous evaluation methods and transparent reporting and governance to enhance the uptake of HTA recommendations and their implementation in practice. Of note, economic evaluation, as part of any framework, is intended to inform rather than prescribe reimbursement decisions. In making decisions, it is vital to consider other essential and relevant value elements that may not be captured in the typical cost-utility analyses, such as equity considerations and other societal benefits. This can be achieved through an augmented CEA approach, multiple-criteria decision analysis (MCDA), or a deliberative approach to decision making [30].

\section{Conclusion}

King Hussein Cancer Center has successfully developed a CET based on the CEA of imatinib in the treatment of CML. The application of the CET in the HTA for cancer medications has allowed for significant reductions in acquisition costs and therefore inclusion of most of the new cancer treatments on the formulary, which would not have been otherwise approved based on their registration prices. A valid, reliable, and relevant CET remains elusive as we strive to strike a balance between opportunity-cost-derived CET and societal and political pressure on the health care system.

Supplementary Information The online version contains supplementary material available at https://doi.org/10.1007/s41669-021-00293-4.

Acknowledgment The authors would like to acknowledge the support of Dr. Asem Mansour, Dr. Hikmat Abdel-Razeq, Dr. Mahmoud AlMasri, Dr. Faris Madanat, and Dr. Faiez Daoud for their support in conducting this research.

\section{Declarations}

Funding Haitham Tuffaha is funded by an NHMRC Early Career Fellowship (GNT1121232).

Conflicts of interest/Competing interests The authors have nothing to disclose in terms of conflicts of interests. 
Availability of data and material Not applicable.

Code availability Not applicable.

Ethics approval King Hussein Cancer Centre Institutional Review Board.

Consent to participate Not applicable.

Consent for publication Not applicable.

Authors' contributions IT conceptualized and drafted the manuscript. All authors critically revised the manuscript and approved the final version.

Open Access This article is licensed under a Creative Commons Attribution-NonCommercial 4.0 International License, which permits any non-commercial use, sharing, adaptation, distribution and reproduction in any medium or format, as long as you give appropriate credit to the original author(s) and the source, provide a link to the Creative Commons licence, and indicate if changes were made. The images or other third party material in this article are included in the article's Creative Commons licence, unless indicated otherwise in a credit line to the material. If material is not included in the article's Creative Commons licence and your intended use is not permitted by statutory regulation or exceeds the permitted use, you will need to obtain permission directly from the copyright holder. To view a copy of this licence, visit http://creativecommons.org/licenses/by-nc/4.0/.

\section{References}

1. American Cancer Society. Global Cancer Facts \& Figures 4th Edition. Published online 2018. https://www.cancer.org/content/ dam/cancer-org/research/cancer-facts-and-statistics/global-cancerfacts-and-figures/global-cancer-facts-and-figures-4th-edition.pdf. Accessed 30 May 2021.

2. Glode AE, May MB. Rising cost of cancer pharmaceuticals: cost issues and interventions to control costs. Pharmacotherapy. 2017;37:85-93.

3. Savage P, Mahmoud S. Development and economic trends in cancer therapeutic drugs: a 5-year update 2010-2014. Br J Cancer. 2015;112:1037-41.

4. Nimri OF, Arqoub K. Statistical Digest Jordan Cancer Registry (JCR) Cancer Incidence in Jordan-2016 Non-Communicable Diseases Directorate-MOH. Publ Online. 2020. https://doi.org/ 10.13140/RG.2.2.31627.92961.

5. Jordan Statistical Yearbook 2017-Department of Statistics. http://dosweb.dos.gov.jo/products/statistical_yearbook2017/. Accessed 9 June 2021.

6. Life expectancy at birth, total (years) - Jordan. https://data.world bank.org/indicator/SP.DYN.LE00.IN?locations $=$ JO\&view $=$ chart. Accessed 1 Mar 2021.

7. World Bank. World Bank. GNI per capita, Atlas method (current US\$)—Jordan. https://data.worldbank.org/indicator/NY.GNP. PCAP.CD ?end $=2018 \&$ locations $=J O \&$ start $=2007 \&$ view $=$ chart . Accessed 1 May 2020.

8. Jordan High Health Council. Jordan National Health Accounts for 2016-2017 Fiscal Years. Published online 2019. http://www.hhc. gov.jo/uploadedimages/\%D9\%85\%D8\%A4\%D8\%B4\%D8\%B1\% D8\%A7\%D8\%AA\%20\%D8\%A7\%D9\%84\%D8\%A7\%D9\%86\% D9\%81\%D8\%A7\%D9\%82\%20\%D8\%A7\%D9\%84\%D8\%B5\% D8\%AD\%D9\%8A\%20.pdf. Accessed 30 May 2021.
9. Abdel-Razeq H, Attiga F, Mansour A. Cancer care in Jordan. Hematol Oncol Stem Cell Ther. 2015;8:64-70.

10. Moe JL, Pappas G, Murray A. Transformational leadership, transnational culture and political competence in globalizing health care services: a case study of Jordan's King Hussein Cancer Center. Global Health. 2007;3:11.

11. Lafi R, Robinson S, Williams I. economic evaluation and the Jordan rational drug list: an exploratory study of national-level priority setting. Value in Health. 2012;15:771-6.

12. Jordan Food and Drug Administration; Medication Pricing Instruction in Jordan. http://jfda.jo/EchoBusV3.0/SystemAssets/ PDF/AR/LawsAndRegulation/Drug/PricingSection. Accessed 1 Mar 2021.

13. Hammad EA. The Use of Economic Evidence to Inform Drug Pricing Decisions in Jordan. Value in Health. 2016;19:233-8.

14. Marseille E, Larson B, Kazi DS, Kahn JG, Rosen S. Thresholds for the cost-effectiveness of interventions: alternative approaches. Bull World Health Organ. 2015;93:118-24.

15. Santos AS, Guerra-Junior AA, Godman B, Morton A, Ruas CM. Cost-effectiveness thresholds: methods for setting and examples from around the world. Expert Rev Pharmacoecon Outcomes Res. 2018;18:277-88.

16. Druker BJ, Guilhot F, O'Brien SG, Gathmann I, Kantarjian H, Gattermann N, et al. Five-year follow-up of patients receiving imatinib for chronic myeloid leukemia. N Engl J Med. 2006;355:2408-17.

17. Jabr M, Treish I. PCASE1 establishment of a cost-effectiveness (CE) threshold at King Hussein Cancer Center: imatinib in chronic myelogenous leukemia (CML). Value in Health. 2008;11:657.

18. Reed SD, Anstrom KJ, Ludmer JA, Glendenning GA, Schulman KA. Cost-effectiveness of imatinib versus interferon-? plus lowdose cytarabine for patients with newly diagnosed chronic-phase chronic myeloid leukemia. Cancer. 2004;101:2574-83.

19. Al-Rabayah A, Jaddoua S. Establishment and implementation of hospital-based health technology assessment at King Hussein Cancer Center: Can our model be an example? Int J Technol Assess Health Care. 2021;37(1):55. https://doi.org/10.1017/S0266 462321000246.

20. Neumann PJ, Cohen JT, Weinstein MC. Updating cost-effectiveness - the curious resilience of the \$50,000-per-QALY threshold. N Engl J Med [Internet]. 2014;371:796-7.

21. Hochhaus A, Larson RA, Guilhot F, Radich JP, Branford S, Hughes TP, et al. Long-Term outcomes of imatinib treatment for chronic myeloid leukemia. N Engl J Med. 2017;376:917-27.

22. Iqbal N, Iqbal N. Imatinib: a breakthrough of targeted therapy in cancer. Chemother Res Pract. 2014;2014:357027.

23. Peggs K, Mackinnon S. Imatinib Mesylate-the new gold standard for treatment of chronic myeloid leukemia. N Engl J Med. 2003;348:1048-50.

24. Timmins, N, Rawlins, M, Appleby, J (eds) A terrible beauty: a short history of NICE, pp. 154-169. http://www.idsihealth.org/ wp-content/uploads/2016/02/A-TERRIBLE-BEAUTY_resize.pdf. Accessed 8 Apr 2020.

25. Claxton K, Martin S, Soares M, Rice N, Spackman E, Hinde S, Devlin N, Smith PC, Sculpher M. Methods for the estimation of the National Institute for Health and Care Excellence cost-effectiveness threshold. Health Technol Assess. 2015;19(14):1-50326.

26. Dillon SA. Carrying NICE over the threshold. NICE. https://www. nice.org.uk/news/blog/carrying-nice-over-the-threshold. Accessed Mar 202021

27. Woods B, Revill P, Sculpher M, Claxton K. Country-level costeffectiveness thresholds: initial estimates and the need for further research. Value Health. 2016;19(8):929-35. 
28. Maraiki F, Byrnes J, Tuffaha H, Hinder M. International HTA experience with targeted therapy approvals for lung cancer. Pharmacoecon Open. 2019;3(1):103-17.

29. Tuffaha HW, Scuffham PA. The Australian managed entry scheme: are we getting it right? Pharmacoeconomics. 2018;36(5):555-65.
30. Phelps CE, Lakdawalla DN, Basu A, Drummond MF, Towse A, Danzon PM. Approaches to aggregation and decision making-a health economics approach: an ISPOR Special Task Force Report. Value Health. 2018;21:146-54. 